\title{
Mutual Influence of the Atmosphere and the Ocean under Wave Processes
}

\author{
Vladimir G. Kirtskhalia' ${ }^{1}$, Konstantin R. Ninidze ${ }^{2}$ \\ ${ }^{1}$ Vekua Sokhumi Institute of Physics and Technology, Tbilisi, Georgia \\ ${ }^{2}$ Sokhumi State University, Tbilisi, Georgia \\ Email: v.kirtskhalia@gmail.com
}

How to cite this paper: Kirtskhalia, V.G. and Ninidze, K.R. (2021) Mutual Influence of the Atmosphere and the Ocean under Wave Processes. Journal of Modern Physics, 12, 1346-1365. https://doi.org/10.4236/jmp.2021.129081

Received: May 30, 2021

Accepted: July 23, 2021

Published: July 26, 2021

Copyright (c) 2021 by author(s) and Scientific Research Publishing Inc. This work is licensed under the Creative Commons Attribution International License (CC BY 4.0).

http://creativecommons.org/licenses/by/4.0/

\begin{abstract}
The article solves the problem of surface gravitational waves using the theory of tangential discontinuity between media: air-water. Using the improved equation of mass continuity and taking into account the atmosphere inhomogeneity in the gravitational field of the Earth, it is shown that during wave processes, these two media mutually influence each other, which explains the reason for the formation of a stormy condition over the ocean and the drop in atmospheric pressure before the storm. The mechanism of the formation of the "killer wave" has been established and thus the "greatest mystery of nature" has been solved. The scale of wind and tsunami wavelengths has been established.
\end{abstract}

\section{Keywords}

Atmosphere, Ocean, Gravitational Waves, Waves of Wind, Tsunami Waves, Killer Wave

\section{Introduction}

In early works (see e.g. [1]), we argued that linear theory describes only capillary waves. As it turned out, this statement was erroneous, but we came to this conclusion thanks to the existing theory of capillary waves, which is based on the assumptions of the potentiality motion of a liquid in the gravitational field of the Earth and its incompressibility. In these assumptions, the linear theory of tangential discontinuity is used in solving the problem, and in the dynamic boundary condition on the liquid surface, the surface tension force is used as the only stabilizing factor of the pressure perturbation ([2], \$62). Nevertheless, in the dispersion equation of capillary waves, a gravitational acceleration is present along with the surface tension coefficient and therefore, these waves are called 
capillary-gravitational. After we showed that the liquid cannot be incompressible, i.e. $\nabla \bar{V} \neq 0 \quad$ [3], where $\vec{V}$-is the velocity of the liquid particle, the gravitational acceleration from the dispersion equation disappeared [1]. This fact led us to believe that gravitational waves should be described by a nonlinear theory. However, a simple analysis shows that in most cases gravitational waves are linear. Indeed, the maximum perturbation of pressure on the water surface in a gravitational wave is equal to $P_{\max }^{\prime}=\rho_{0} g a$, where $a$ is the amplitude of the wave and $\rho_{0}$ is the undisturbed density of water. At $a=3 \mathrm{~m}$, which is approximately equal to the maximum value of the amplitude of the wind wave away from the coast and is greater than the amplitude of the tsunami wave, this disturbance is equal to $2.7 \times 10^{4} \mathrm{~Pa}$, while the equilibrium pressure is $P_{0}=1 \mathrm{~atm} .=1.013 \times 10^{5}$ $\mathrm{Pa}$. Thus, the pressure on the surface of water can be represented in the following form $P=P_{0}+P^{\prime}$, where $P^{\prime} / P_{0}<1$, and therefore, linear theory can be used.

A surface gravitational wave is generated and propagated at the interface between two media, i.e. it is a typical problem of tangential discontinuity. Therefore, the dispersion equation must contain the thermodynamic parameters of both media. Despite this, when solving this problem, many authors do not take into account the influence of atmospheric parameters on the phenomenon under study (see for example. [3] [4] [5]), but nevertheless, they get results that match the results of observations. For example, in work [3] the two-dimensional problem of the surface gravitational wave was solved, in which the following are used:

1) Equation of motion of fluid $-\rho \frac{\mathrm{d} \vec{V}}{\mathrm{~d} t}=-\nabla P+\rho \vec{g}$

2) Equation of incompressibility of liquid $-\nabla \vec{V}=0$

Here: $\vec{V}$ is velocity of a liquid particle, $P$-pressure, $\rho$-density, $\vec{g}$ - gravitational acceleration. It is assumed that the velocity is small and after linearization, the system of Equations (1), (2) takes the form:

$$
\begin{gathered}
\frac{\partial u}{\partial t}=-\frac{1}{\rho} \frac{\partial P}{\partial x}, \\
\frac{\partial w}{\partial t}=-\frac{1}{\rho} \frac{\partial P}{\partial z}-g, \\
\frac{\partial u}{\partial x}+\frac{\partial w}{\partial z}=0 .
\end{gathered}
$$

By denoting $\xi(x, t)=\xi_{0} \cos (\omega t-k x)$, the displacement of the liquid surface along the $Z$ axis and writing the boundary conditions on the free surface and on the bottom as

$$
\left.w\right|_{z=0}=\frac{\partial \xi}{\partial t} \text { and }\left.w\right|_{z=-H}=0,
$$

solutions of the system of Equations (3), (4), (5) will be: 


$$
\begin{gathered}
u=\xi_{0} \omega \frac{\cosh [k(z+H)]}{\sinh (k H)} \cos (\omega t-k x), \\
w=-\xi_{0} \omega \frac{\sinh [k(z+H)]}{\sinh (k H)} \sin (\omega t-k x), \\
P=-\rho g z+\rho g \xi_{0} \frac{\cosh [k(z+H)]}{\cosh (k H)} \cos (\omega t-k x) .
\end{gathered}
$$

Substituting solutions (7) and (9) in (3), or (8) and (9) in (4) at, the author obtains an expression for the phase velocity in the form:

$$
\left|U_{p}\right|=\sqrt{\frac{g}{k} \tanh (k H)} .
$$

For deep water, when $k H=2 \pi H / \lambda>1 \Rightarrow \tanh (k H) \cong 1$, where $\lambda$ is the wavelength, from (10) follows

$$
\left|U_{p}\right|=\sqrt{\frac{g}{k}}
$$

and for shallow water, when $k H=2 \pi H / \lambda<1 \Rightarrow \tanh (k H) \cong k H$,

$$
\left|U_{p}\right|=\sqrt{g H} .
$$

Similar results were obtained in [4] [5] using the hydrostatic approximation method, in which Equation (4) is integrated under the following assumption $\partial w / \partial t=0$. In the work [3] it is noted that the validity of this assumption is not proven and this doubt is well-founded, because firstly- $\partial w / \partial t=0$ means that $w=$ const $=0$, and therefore, no vertical movement of the liquid particle occurs, and second-at $\partial w / \partial t=0$, from Equation (4) follows $\nabla P=\rho \vec{g}$ which is a condition for the equilibrium of the liquid and therefore, vibrations are impossible.

As noted above, while analyzing the problem of surface gravity waves, both media should be taken into account-air and water, i.e. should be obtained in a linear approximation of the equations of gravitational waves in these media, and then, tied to their solutions on the boundary surface using boundary conditions.

When performing these procedures, the perturbed values of density and pressure in each medium are linked by the equation of the state of the medium $\rho=P^{\prime} / C^{2}$, where $C$ is the speed of sound in the medium. We called attention to the absurdity of the fact that the speed of sound in the entire atmosphere is calculated by the formula $C=\sqrt{\gamma k_{B} T / m_{0}}$ [6] [7], where $\gamma=c_{p} / c_{v}=1.4$ is an adiabatic index of air and is equal to the ratio of heat capacities at constant pressure and volume, $k_{B}=1.38 \times 10^{-23} \mathrm{~J} / \mathrm{K}$-the Boltzmann constant, $m_{0}=4.81 \times 10^{-26} \mathrm{~kg}$-mass of one air molecule, $T$-absolute temperature. This means that on the altitude of $60 \mathrm{~km}$ and on the North Pole, where the temperatures are the same and equal to $-40^{\circ} \mathrm{C}$, the speeds of sounds should have the same values.

We have found the answer to this paradox and it is that the abovementioned formula is just only for a homogeneous medium where the density depends only 
on the pressure $\rho=\rho(P)$. In the inhomogeneous medium, which is the earth's atmosphere, due to the influence of the gravitational field on it, the density also depends on entropy $\rho=\rho(P, S)$. In that case the density perturbation equals to the following:

$$
\rho^{\prime}=\left(\frac{\partial \rho_{0}}{\partial P_{0}}\right)_{s} P^{\prime}+\left(\frac{\partial \rho_{0}}{\partial S_{0}}\right)_{p} S^{\prime},
$$

where $P_{0}, \rho_{0}, S_{0}$-represent unperturbed values of pressure, density and entropy accordingly. Considering that $S^{\prime}=\left(\partial S_{0} / \partial P_{0}\right)_{T} P^{\prime}$, from (13) we easily receive [8] [9]:

$$
\rho^{\prime}=\left(\frac{1}{C_{s}^{2}}+\frac{1}{C_{p}^{2}}\right) P^{\prime}=\frac{1}{C^{2}} P^{\prime} .
$$

Here:

$$
\begin{gathered}
C_{S}^{2}=\left(\frac{\partial P_{0}}{\partial \rho_{0}}\right)=\gamma \frac{k_{B} T}{m_{0}} \text { is the speed of adiabatic sound, } \\
C_{P}^{2}=\left[\left(\frac{\partial \rho_{0}}{\partial S_{0}}\right)_{P}\left(\frac{\partial S_{0}}{\partial P_{0}}\right)_{T}^{-1}=\frac{c_{p} \rho_{0}^{2}}{T\left(\partial \rho_{0} / \partial T\right)_{s}^{2}}\right. \text { is the speed of isobaric sound, } \\
C^{2}=\frac{C_{s}^{2} C_{p}^{2}}{C_{s}^{2}+C_{p}^{2}} \text { is the true value of the speed of sound. }
\end{gathered}
$$

Thus, the square of the true value of the speed of sound is reduced from the squares of the velocities of adiabatic and isobaric sounds. Substituting into (16) the value $\rho_{0}$ from the Laplace equation

$$
\rho_{0}=\rho_{0}^{0} \exp \left(-m_{0} g z / k_{B} T\right),
$$

where $\rho_{0}^{0}$-is unperturbed density value at sea level, for the speed of isobaric sound we receive the following:

$$
C_{p}=\sqrt{\frac{c_{p} k_{B}^{2} T^{3}}{m_{0}^{2} g^{2} z^{2}}} .
$$

Using (15) and (19), from (17) we find:

$$
C(z, T)=\sqrt{\frac{\gamma k_{B} T}{m_{0}\left(1+\frac{\gamma m g^{2} z^{2}}{c_{p} k_{B} T^{2}}\right)}} .
$$

We can see that the true value of the speed of sound in the Earth's atmosphere truly depends on the altitude (density) and condition $z=0$ is equivalent to the condition $g=0$. Thus, at the sea level, air is a homogeneous medium and the speed of sound should be calculated by the Equation (15).

Figure 1 shows the temperature distribution over height in the Earth's atmosphere [10]. We can see that up to the altitude of approximately $11 \mathrm{~km}$., the temperature drops according to strictly linear law $T=\alpha+\beta z$, where $\alpha=288.15 \mathrm{~K}$ 


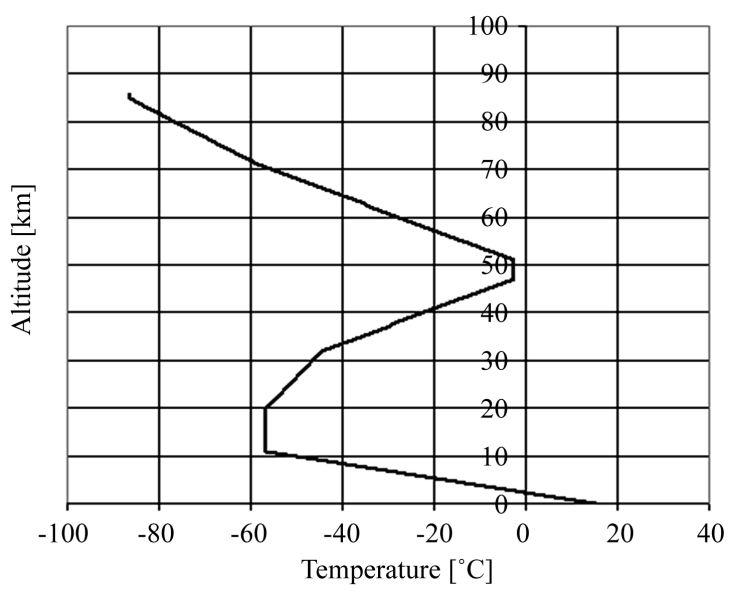

Figure 1. Temperature as a function of geometrical altitude.

and $\beta=-6.52 \times 10^{-3} \mathrm{~K} / \mathrm{m}$. This part of the atmosphere is called the troposphere, which has no heat source, hence the entropy $S$ is constant, i.e. can be used the adiabatic equation

$$
\frac{\mathrm{d} S}{\mathrm{~d} t}=\frac{\partial S}{\partial t}+(\vec{V} \nabla) S=0,
$$

On the top boundary of the troposphere, the drop in temperature abruptly stops and remains constant till the altitude of approximately $20 \mathrm{~km}$ (tropopause), and then increases in the stratosphere. In this regard, the law of entropy constancy, which was used in our calculations, ceases to be true. Therefore, our theory is valid up to the height $z \cong 11 \mathrm{~km}$.

Substituting in (15), (19) and (20) $T=288.15-6.53 \times 10^{-3} z$ and taking into account that $c_{p}=10^{3} \mathrm{~J} / \mathrm{kg} \cdot \mathrm{K}$, we get:

$$
\begin{gathered}
C_{s}(z)=\sqrt{401.66\left(288.15-6.53 \times 10^{-3} z\right)}, \\
C_{p}(z)=925.70 \frac{\sqrt{\left(288.15-6.53 \times 10^{-3} z\right)^{3}}}{z}, \\
C(z)=20.05 \sqrt{\frac{\left(288.15-6.53 \times 10^{-3} z\right)^{3}}{\left(288.15-6.53 \times 10^{-3} z\right)^{2}+4.69 \times 10^{-4} z^{2}}} .
\end{gathered}
$$

In Figure 2, the graphs of expressions (22) and (23) are shown. Calculations show that the relative inaccuracy, between values $C_{s}(z)$ and $C(z)$ at heights $z=1 \mathrm{~km}$ and $z=10 \mathrm{~km}$ is equal to $0.3 \%$ and $33 \%$, respectively, i.e. increases 110 times. Obviously, such an error cannot be ignored when calculating the Mach number [11].

In the work [12], the author suggests that at the upper boundary of the troposphere ( $z \cong(10-11) \mathrm{km}$ which is often called the ozone layer), there is an exothermic reaction of ozone synthesis $\left(\mathrm{O}_{2}+\mathrm{O} \rightarrow \mathrm{O}_{3}+24 \mathrm{k} . \mathrm{cal} / \mathrm{mol}\right)$, which is the reason for such dynamics of the temperature distribution. Let us show that our theory fully confirms this hypothesis. 


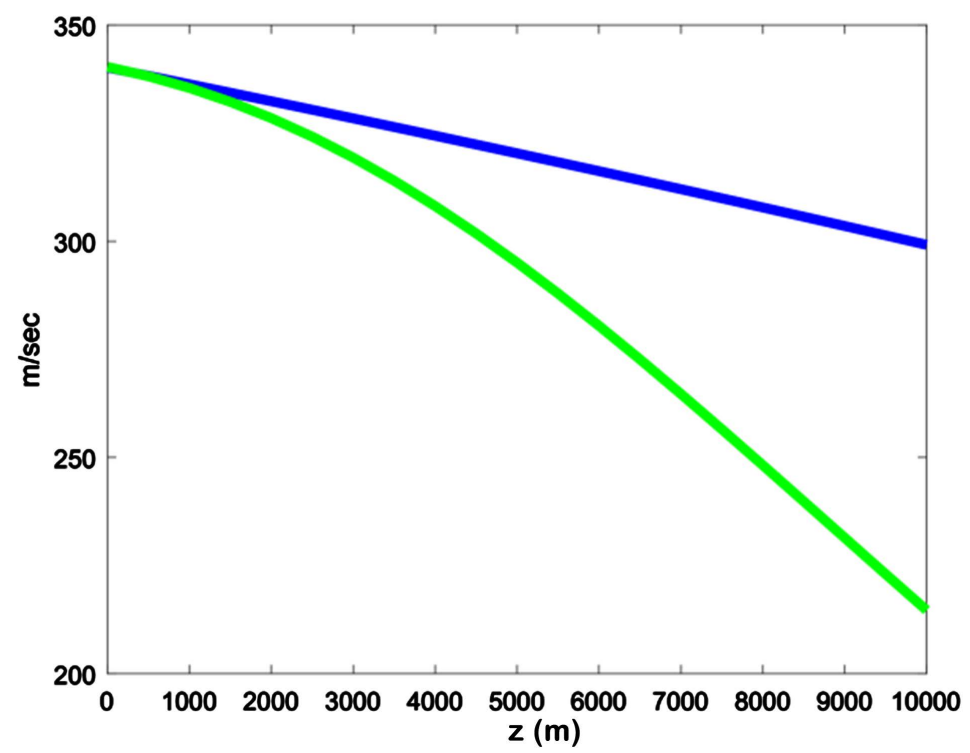

Figure 2. Dependence of isobaric $\left(C_{s}(z)\right.$-the blue curve) and true $(C(z)$-the green curve) speeds of sounds on the altitude in the troposphere.

In Figure 3, the graphs of the height distribution of the adiabatic $C_{s}(z)$ and isobaric $C_{p}(z)$ velocities of sounds are shown. It is seen that these graphs intersect at a height of $z \cong 10200 \mathrm{~m}$. Equating $C_{s}^{2}(T)$ and $C_{p}^{2}(z, T)$ from Formulas (15) and (19), we obtain

$$
\frac{\gamma k T}{m_{0}}=\frac{c_{p} k^{2} T^{3}}{m_{0}^{2} g^{2} z^{2}} \Rightarrow z=\sqrt{\frac{c_{p} k}{\gamma m_{0}}} \frac{T}{g} .
$$

Substituting in (25) $T=\alpha+\beta z$ we find:

$$
z=\frac{\sqrt{c_{p} k \alpha^{2} / \gamma m_{0} g^{2}}}{1+\sqrt{c_{p} k \beta^{2} / \gamma m_{0} g^{2}}}=10230 \mathrm{~m} .
$$

This altitude almost exactly coincides with the upper boundary of the troposphere, presented in Figure 1, which proves the high reliability of our theory. Thus, it can be said with high confidence, that expressions (25) and/or (26) are equations of the upper border of the troposphere, where adiabatic and isobaric speeds of sound are equated, i.e. a resonance $\omega_{s}=\omega_{p}$ occurs. As it is known, the abrupt change in the dynamics of the process, which is observed in Figure 1, is as a general rule in connection with resonance. Thus, it can be assumed that the resonance of frequencies of the adiabatic and isobaric sounds is a trigger mechanism for the exothermic reaction of ozone synthesis and, as a consequence, the release of a large amount of heat.

The discovery of an isobaric sound leads to another important result. Let us transform the following expression:

$$
\left[\frac{\rho_{0}}{\left(\partial \rho_{0} / \partial T\right)_{p}}\right]^{2}=\left[\frac{m}{v}\left(\frac{\partial(m / v)}{\partial T}\right)_{p}^{-1}\right]_{m=\text { const }}^{2}=\left[\frac{1}{v}\left(\frac{\partial v}{\partial T}\right)_{p}\right]^{-2}=\frac{1}{\beta_{p}^{2}},
$$




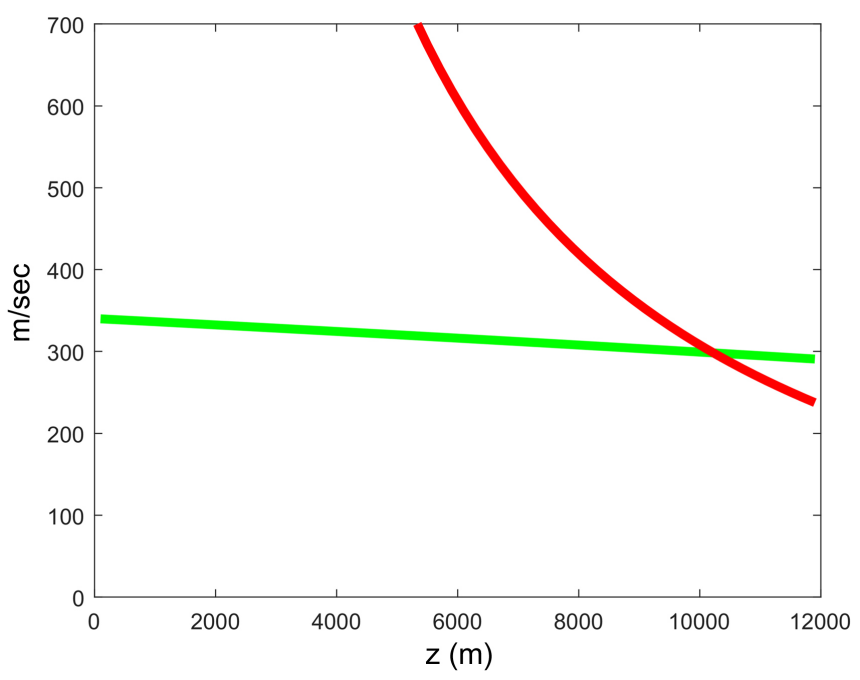

Figure 3. The dependences of adiabatic ( $C_{s}(T)$-green curve) and isobaric ( $C_{p}(z, T)$-red curve) sound velocities from the altitude in the troposphere.

where $v$-is gas volume and $\beta_{p}$-is the coefficient of thermal expansion. Then from (16) we shall find that:

$$
C_{p}(z, T)=\frac{1}{\beta_{p}}\left(\frac{c_{p}}{T}\right)^{1 / 2} .
$$

From (28) it is derived, that $\beta_{p}=\beta_{p}(z, T)$, i.e. the coefficient of the thermal expansion depends not only on the temperature, as is usual to modern thermodynamics, but also on the altitude of the atmosphere. This fact invalidates the universality of the laws of the ideal gas. More detailed account of this please see the work [13].

Since the sound wave carries the density perturbation, an idea of generalization of the equation of mass continuity for an inhomogeneous medium arose. This equation was also received for the homogeneous medium and it determines the change of density as a result of substance mass change in the constant volume. However, the change in density is possibly also due to the change of volume of the constant mass of the substance, i.e.:

$$
\frac{\mathrm{d} \rho}{\mathrm{d} t}=\frac{\mathrm{d}}{\mathrm{d} t}\left(\frac{m}{v}\right)=\frac{v \frac{\mathrm{d} m}{\mathrm{~d} t}-m \frac{\mathrm{d} v}{\mathrm{~d} t}}{v^{2}}=\left(\frac{\mathrm{d} \rho}{\mathrm{d} t}\right)_{v}-\left(\rho \frac{\mathrm{d}}{\mathrm{d} t} \ln v\right)_{m},
$$

Equation (29) is reduced to the following [14]:

$$
\frac{\mathrm{d} \rho}{\mathrm{d} t}=-\rho \nabla \vec{V}-\frac{\vec{V} \nabla P}{C_{p}^{2}} .
$$

The first summand in the right side of the Equation (30) determines the change of mass in the constant volume while the second summand is the isobaric change of the volume of the substance constant mass as a result of the change in temperature, which is caused by the change in entropy in the inhomogeneous medium. This work has radically altered the existing concepts of compressibility 
and incompressibility of medium. It is considered, that these concepts have a mechanical meaning. In reality, they have thermodynamic meaning and characterize the homogeneity or non homogeneity of the medium. The homogeneous medium is always compressible. The incompressibility is a consequence of its non homogeneity and it manifests as strongly as inhomogeneous the medium is, as it occurs in the Earth's atmosphere with increasing altitude. The speed of sound in a homogeneous medium is adiabatic, and in an inhomogeneous medium it is a combination of the speeds of adiabatic and isobaric sounds.

The work [1] considers the problem of internal waves from the monograph [2]. The authors consider the water to be incompressible and contemplate, that the density perturbation is isobaric, i.e. only the second summand is considered in the Equation (13). As a result, they receive a so-called internal wave, the frequency of which depends only on the direction of the wave vector, the magnitude of which can be any. It is clear that such wave does not exist in nature and the reason for this paradox is the use of incompressibility condition towards the water. Hence, water is a compressible (homogeneous) medium. Though it may seem paradoxical, water is a much more compressible medium (in the thermodynamic sense), than the atmosphere in the higher layers. Work [14] has been dedicated to this problem.

Another paradox that we noticed is that the Euler equation in its current form contradicts the basic principle of physics. Indeed, let us consider the Euler equation in its generally accepted form: $\rho \mathrm{d} \vec{V} / \mathrm{d} t=\rho[\partial \vec{V} / \partial t+(\vec{V} \nabla) \vec{V}]=-\nabla P+\rho \vec{g}$. If $\vec{V}_{0}$ there is a stationary velocity of motion of the liquid and $\vec{V}^{\prime}$ is its small perturbation, then after linearization its left side, which determines the acceleration of the liquid particle, will be equal to $\left[\partial \vec{V}^{\prime} / \partial t+\left(\vec{V}_{0} \nabla\right) \vec{V}^{\prime}\right]$. Thus, the acceleration of a liquid particle depends on the stationary velocity of the medium, which contradicts the principle of relativity. This contradiction is caused by the assumption $\mathrm{d} \rho / \mathrm{d} t=0 \Rightarrow \rho=$ const, i.e. the assumption of incompressibility of the liquid $\nabla \vec{V}=0$ and neglect of the second term in Equation (30). In fact, if the first term is equal to zero (incompressible medium), remains the second term, and if the second term is equal to zero (compressible medium), the first remains. Thus, $\mathrm{d} \rho / \mathrm{d} t \neq 0$ and density $\rho$ must be entered under the derivative

$$
\frac{\mathrm{d}(\rho \vec{V})}{\mathrm{d} t}=\vec{V} \frac{\mathrm{d} \rho}{\mathrm{d} t}+\rho \frac{\mathrm{d} \vec{V}}{\mathrm{~d} t}=-\nabla P+\rho \vec{g} .
$$

Let's substitute in the right side of Equation (31) the value $\mathrm{d} \rho / \mathrm{d} t$ from Equation (30)

$$
\begin{aligned}
& -\vec{V} \rho \nabla \vec{V}-\frac{V^{2} \nabla P}{C_{p}^{2}}+\rho \frac{\mathrm{d} \vec{V}}{\mathrm{~d} t}=-\nabla P+\rho \vec{g} \\
& \Rightarrow \rho\left[\frac{\mathrm{d} \vec{V}}{\mathrm{~d} t}-(V \nabla) \dot{V}\right]-\frac{V^{2} \nabla P}{C_{p}^{2}}=-\nabla P+\rho \vec{g}
\end{aligned}
$$

Neglect the term $V^{2} \nabla P / C_{p}^{2}$, the Euler equation takes the form:

$$
\rho \frac{\partial \vec{V}}{\partial t}=-\nabla P+\rho \vec{g}
$$


As can be seen the nonlinear term dropped out of the Euler equation and, therefore, there is no contradiction. The Equation (30) along with the Equation (27) was used by us in the problem of the capillary waves [15], after which all existing contradictions were removed.

In our opinion, plasma is an incompressible medium, because it is a substantially inhomogeneous, due to the fact that each electron and ion are in a force field created by neighboring particles. It can be assumed that this is precisely the reason for the difficulties in implementing a controlled thermonuclear reaction, since it is impossible to compress the plasma to the required state and keep it in this state for a long enough period of time.

\section{A New Approach to the Theory of Surface Gravitational Waves}

Let us now apply these equations to the problem of surface gravitational waves:

$$
\left\{\begin{array}{l}
\rho \frac{\mathrm{d} \vec{V}}{\mathrm{~d} t}=\rho\left[\frac{\partial \vec{V}}{\partial t}+(\vec{V} \nabla) \vec{V}\right]=-\nabla P+\rho \vec{g} \\
\frac{\mathrm{d} \rho}{\mathrm{d} t}=\frac{\partial \rho}{\partial t}+(\vec{V} \nabla) \rho=-\rho \nabla \vec{V}-\frac{\vec{V} \nabla P}{C_{p}^{2}}
\end{array}\right.
$$

Suppose that $V_{0}=0$ and let's represent all the variables as the sum of their stationary values and small perturbations:

$$
P=P_{0}+P^{\prime} ; \rho=\rho_{0}+\rho^{\prime} ; \vec{V}=\vec{V}^{\prime} .
$$

Using under the linearization of system (34) the condition of equilibrium of the medium in the gravitational field of the Earth $\nabla P_{0}=\rho_{0} \vec{g}$, which is true for both air and water, we get:

$$
\left\{\begin{array}{l}
\rho_{0} \frac{\partial \vec{V}^{\prime}}{\partial t}=-\nabla P^{\prime}+\rho^{\prime} \vec{g} \\
\frac{\partial \rho^{\prime}}{\partial t}+\left(\vec{V}^{\prime \prime} \nabla\right) \rho_{0}=-\rho_{0} \nabla \vec{V}^{\prime}-\frac{\rho_{0} \vec{g} \vec{V}^{\prime}}{C_{p}^{2}}
\end{array}\right.
$$

Let's apply the operator $\nabla$ to the first equation of the system (32) and the operator $\partial / \partial t$ to the second, after which we have

$$
\left\{\begin{array}{l}
\nabla \rho_{0} \frac{\partial \vec{V}^{\prime}}{\partial t}+\rho_{0} \nabla \frac{\partial \vec{V}^{\prime}}{\partial t}=-\Delta P^{\prime}+\nabla \rho^{\prime} \vec{g} \\
\frac{\partial^{2} \rho^{\prime}}{\partial t^{2}}+\left(\frac{\partial \vec{V}^{\prime}}{\partial t} \nabla\right) \rho_{0}=-\rho_{0} \nabla \frac{\partial \vec{V}^{\prime}}{\partial t}-\frac{\partial \vec{V}^{\prime}}{\partial t} \frac{\nabla P_{0}}{C_{p}^{2}}
\end{array}\right.
$$

Given that $P_{0}$ and $\rho_{0}$ in air and in water depend only on the vertical $Z$ coordinate, the following relations are valid:

$$
z \nabla \rho_{0} \frac{\partial \vec{V}^{\prime}}{\partial t}=\left(\frac{\partial \vec{V}^{\prime}}{\partial t} \nabla\right) \rho_{0}=\frac{\mathrm{d} \rho_{0}}{\mathrm{~d} z} \frac{\partial V_{z}^{\prime}}{\partial t} ; \frac{\partial \vec{V}^{\prime}}{\partial t} \frac{\nabla P_{0}}{C_{p}^{2}}=-\frac{\rho_{0} g}{C_{p}^{2}} \frac{\partial V_{z}^{\prime}}{\partial t} \nabla \rho^{\prime} \vec{g}=-g \frac{\partial \rho^{\prime}}{\partial z},
$$

after that, the system (36) can be rewritten as 


$$
\left\{\begin{array}{l}
\frac{\mathrm{d} \rho_{0}}{\mathrm{~d} z} \frac{\partial V_{z}^{\prime}}{\partial t}+\rho_{0} \nabla \frac{\partial \vec{V}^{\prime}}{\partial t}=-\Delta P^{\prime}-g \frac{\partial \rho^{\prime}}{\partial z} \\
\frac{\mathrm{d} \rho_{0}}{\mathrm{~d} z} \frac{\partial V_{z}^{\prime}}{\partial t}+\rho_{0} \nabla \frac{\partial \vec{V}^{\prime}}{\partial t}=-\frac{\partial^{2} \rho^{\prime}}{\partial t^{2}}+\frac{\rho_{0} g}{C_{p}^{2}} \frac{\partial V_{z}^{\prime}}{\partial t}
\end{array}\right.
$$

Equating the right parts of the system (37) we get

$$
\Delta P^{\prime}+g \frac{\partial \rho^{\prime}}{\partial z}+\frac{\rho_{0} g}{C_{p}^{2}} \frac{\partial V_{z}^{\prime}}{\partial t}-\frac{\partial^{2} \rho^{\prime}}{\partial t^{2}}=0 .
$$

Using the equation of state of the medium $\rho^{\prime}=\left(1 / C^{2}\right) P^{\prime}$ and expressing $\partial V_{z}^{\prime} / \partial t$ from the first equation of the system (35) $\partial V_{z}^{\prime} / \partial t=-\left(1 / \rho_{0}\right)\left(\partial P^{\prime} / \partial z+g P^{\prime} / C^{2}\right)$, Equation (38) takes the form:

$$
\Delta P^{\prime}+g \frac{\partial}{\partial z}\left(\frac{P^{\prime}}{C^{2}}\right)-\frac{g}{C_{p}^{2}}\left(\frac{\partial P^{\prime}}{\partial z}+g \frac{P^{\prime}}{C^{2}}\right)-\frac{1}{C^{2}} \frac{\partial^{2} P^{\prime}}{\partial t^{2}}=0 .
$$

$C$-is the speed of sound in the gravitational field of the Earth and is an important thermodynamic parameter of the medium, which, like other parameters, must depend on the vertical $z$ coordinate. It is expressed in terms of the adiabatic $C_{s}$ and isobaric $C_{p}$ speeds of sounds by the following ratio [8]:

$$
\frac{1}{C^{2}(z)}=\frac{1}{C_{s}^{2}(z)}+\frac{1}{C_{p}^{2}(z)} \Rightarrow C^{2}(z)=\frac{C_{s}^{2}(z) C_{p}^{2}(z)}{C_{s}^{2}(z)+C_{p}^{2}(z)} .
$$

Taking into account (40), Equation (39) can be written as:

$$
\Delta P^{\prime}+\frac{g}{C_{s}^{2}(z)} \frac{\partial P^{\prime}}{\partial z}-\frac{g}{C^{2}(z)}\left(\frac{1}{C^{2}(z)} \frac{\mathrm{d} C^{2}(z)}{\mathrm{d} z}+\frac{g}{C_{p}^{2}(z)}\right) P^{\prime}-\frac{1}{C^{2}(z)} \frac{\partial^{2} P^{\prime}}{\partial t^{2}}=0 .
$$

We introduce the notation:

$$
\begin{aligned}
& \Gamma(z)=\frac{1}{C^{4}(z)} \frac{\mathrm{d} C^{2}(z)}{\mathrm{d} z}=\frac{1}{C_{s}^{4}(z)} \frac{\mathrm{d} C_{s}^{2}(z)}{\mathrm{d} z}+\frac{1}{C_{p}^{4}(z)} \frac{\mathrm{d} C_{p}^{2}(z)}{\mathrm{d} z} ; \\
& \Sigma(z)=\frac{1}{C^{2}(z) C_{p}^{2}(z)}
\end{aligned}
$$

and then, Equation (39) finally takes the form:

$$
\Delta P^{\prime}+\frac{g}{C_{s}^{2}(z)} \frac{\partial P^{\prime}}{\partial z}-g[\Gamma(z)+g \Sigma(z)] P^{\prime}-\frac{1}{C^{2}(z)} \frac{\partial^{2} P^{\prime}}{\partial t^{2}}=0 .
$$

Equation (43) is the equation of mechanical waves in any medium in the earth's gravitational field, or the generalized equation of gravitational waves. We will search $P^{\prime}$ in the form:

$$
P^{\prime}(x, z, t)=P_{a}(z) \exp [i(k x-\omega t)]
$$

after then from (43) we get:

$$
\frac{\mathrm{d}^{2} P_{a}(z)}{\mathrm{d} z^{2}}+\frac{g}{C_{s}^{2}(z)} \frac{\mathrm{d} P_{a}(z)}{\mathrm{d} z}-\left\{k^{2}+g[\Gamma(z)+g \Sigma(z)]-\frac{\omega^{2}}{C^{2}(z)}\right\} P_{a}^{\prime}(z)=0 .
$$

Let's denote the values related to air $(z>0)$ by index 1 , and to water 
$(z<0)$-by index 2 and consider this equation for air, where:

$$
C_{s 1}^{2}=\gamma \frac{k_{B} T}{m_{0}} \text { and } C_{p 1}^{2}=\frac{c_{p} k_{B}^{2} T^{3}}{m_{0}^{2} g^{2} z^{2}} .
$$

Substituting in (46) $T=\alpha+\beta z$, where $\alpha=288.15 \mathrm{~K}$ and $\beta=-6.52 \times 10^{-3} \mathrm{~K} / \mathrm{m}$, we get:

$$
C_{s 1}^{2}(z)=\gamma \frac{k_{B}(\alpha+\beta z)}{m_{0}} \text { and } C_{p 1}^{2}(z)=\frac{c_{p} k_{B}^{2}(\alpha+\beta z)^{3}}{m_{0}^{2} g^{2} z^{2}} .
$$

Thus, Equation (45) is a second-order differential equation with variable coefficients. Considering that $c_{p}=10^{3} \mathrm{~J} / \mathrm{kg} \cdot \mathrm{K}$, to simplify the problem, we will average these coefficients in the range of heights from $z=z_{0}=10000 \mathrm{~m}$ after which the following relations are valid

$$
\begin{gathered}
\frac{1}{\bar{C}_{s 1}^{2}}=\frac{1}{z_{0}} \int_{0}^{z_{0}} \frac{1}{C_{s 1}^{2}(z)} \mathrm{d} z=9.80 \times 10^{-6} \frac{\mathrm{sec}^{2}}{\mathrm{~m}^{2}}, \\
\frac{1}{\bar{C}_{p 1}^{2}}=\frac{1}{z_{0}} \int_{0}^{z_{0}} \frac{1}{C_{p 1}^{2}(z)} \mathrm{d} z=2.90 \times 10^{-6} \frac{\mathrm{sec}^{2}}{\mathrm{~m}^{2}}, \\
\frac{1}{\bar{C}_{1}^{2}}=\frac{1}{z_{0}} \int_{0}^{z_{0}} \frac{1}{C_{1}^{2}(z)} \mathrm{d} z=12.70 \times 10^{-6} \frac{\mathrm{sec}^{2}}{\mathrm{~m}^{2}}, \\
\bar{\Gamma}_{1}=\frac{1}{z_{0}} \int_{0}^{z_{0}} \Gamma(z) \mathrm{d} z=-1.30 \times 10^{-9} \frac{\mathrm{sec}^{2}}{\mathrm{~m}^{3}}, \\
\bar{\Sigma}_{1}=\frac{1}{z_{0}} \int_{0}^{z_{0}} \Sigma_{1}(z) \mathrm{d} z=4.75 \times 10^{-11} \frac{\mathrm{sec}^{4}}{\mathrm{~m}^{4}} .
\end{gathered}
$$

Denoting $g\left(\bar{\Gamma}_{1}+g \bar{\Sigma}_{1}\right)=\bar{\Omega}_{1}=-8.19 \times 10^{-9} \mathrm{~m}^{-2}$, Equation (45) for air takes the form:

$$
\frac{\mathrm{d}^{2} P_{a 1}(z)}{\mathrm{d} z^{2}}+\frac{g}{\bar{C}_{s 1}^{2}} \frac{\mathrm{d} P_{a 1}(z)}{\mathrm{d} z}-\left(k^{2}+\bar{\Omega}_{1}-\frac{\omega^{2}}{\bar{C}_{1}^{2}}\right) P_{a 1}(z)=0 .
$$

We will seek a solution to Equation (49) in the form

$$
P_{a 1}(z)=A \exp (\gamma z),
$$

which gives

$$
P_{a 1}(z)=A_{1} \exp \left(\gamma_{1} z\right)+A_{2} \exp \left(\gamma_{2} z\right)
$$

where:

$$
\begin{aligned}
& \gamma_{1}=-\frac{k}{\theta_{s 1}}\left[1+\sqrt{1+\theta_{s 1}^{2}\left(1+\frac{\bar{\Omega}_{1}}{k^{2}}-x^{2}\right)}\right], \\
& \gamma_{2}=-\frac{k}{\theta_{s 1}}\left[1-\sqrt{1+\theta_{s 1}^{2}\left(1+\frac{\bar{\Omega}_{1}}{k^{2}}-x^{2}\right)}\right] .
\end{aligned}
$$

Here $x=U_{p} / \bar{C}_{1}$ and $U_{p}=\omega / k$-is the phase velocity of the wave. $\theta_{s 1}$ -dimensionless quantity that is equal to 


$$
\theta_{s 1}=\frac{2 k \bar{C}_{s 1}^{2}}{g}
$$

It is easy to see that if the condition is met

$$
1+\theta_{s 1}^{2}\left(1+\frac{\bar{\Omega}_{1}}{k^{2}}-x^{2}\right)>1
$$

we have: $\gamma_{1}<0, \gamma_{2}>0$ and then, based on the wave surface condition $\left(P_{a 1}(z) \rightarrow 0\right.$ when $\left.z \rightarrow \infty\right)$, in (51) must be put $A_{2}=0$. On condition

$$
0 \leq 1+\theta_{s 1}^{2}\left(1+\frac{\bar{\Omega}_{1}}{k^{2}}-x^{2}\right)<1,
$$

we have: $\gamma_{1}<0, \gamma_{2}<0$ a and then in the right part (51), both terms should be taken into account. Substituting the value $\bar{\Omega}_{1}=-8.19 \times 10^{-9} \mathrm{~m}^{-2}$, the solutions of inequalities (56) and (56) are:

$$
\begin{gathered}
k>9.05 \times 10^{-5} / \sqrt{1-x^{2}} \mathrm{~m}^{-1} \Rightarrow \lambda<6.90 \times 10^{4} \sqrt{1-x^{2}} \mathrm{~m}, \\
k<9.05 \times 10^{-5} / \sqrt{1-x^{2}} \mathrm{~m}^{-1} \Rightarrow \lambda>6.90 \times 10^{4} \sqrt{1-x^{2}} \mathrm{~m} .
\end{gathered}
$$

let's call the waves satisfying condition (57) wind waves, and condition (58) tsunami waves.

For water $(z<0)$ dependencies $C_{s 2}$ and $C_{p 2}$ on $z$ are unknown, but we can assume with a high probability that they are very weak. As for their numerical values, they can be determined from the experimental data. Substituting in (28) the values of the coefficient of thermal expansion and specific heat capacity at constant pressure for water $\beta_{p}=1.50 \times 10^{-4} \mathrm{~K}^{-1}, \quad c_{p}=4.19 \times 10^{3} \mathrm{~J} / \mathrm{kg} \cdot \mathrm{K}$ at a temperature of $T=288 \mathrm{~K}$ we obtain $C_{p 2}=25210 \mathrm{~m} / \mathrm{sec}$. On the other hand, the speed of sound in water, measured experimentally with great accuracy $C_{2}=1480 \mathrm{~m} / \mathrm{sec}$, and then, from formula (17), we have $C_{s 2}=C_{2} C_{p 2} / \sqrt{C_{p 2}^{2}-C_{2}^{2}}=1482.60 \mathrm{~m} / \mathrm{sec}$. As we can see, the speed of sound in water is practically equal to the adiabatic speed of sound, i.e. $C_{2}=C_{s 2}$. This result irrefutably proves that the mechanism of sound propagation in water is adiabatic $\left(C_{p 2}=\infty\right)$ and on the right-hand side of Equation (30) only the first term remains. Thus, Equation (45) for water has the form:

$$
\frac{\mathrm{d}^{2} P_{a 2}(z)}{\mathrm{d} z^{2}}+\frac{g}{C_{2}^{2}} \frac{\mathrm{d} P_{a 2}(z)}{\mathrm{d} z}-\left(k^{2}-\frac{\omega^{2}}{C_{2}^{2}}\right) P_{a 2}(z)=0
$$

Representing the amplitude of the pressure perturbation in the form $P_{a 2}(z)=B \exp (\delta z)$ from (59), we obtain

$$
P_{a 2}(z)=B_{1} \exp \left(\delta_{1} z\right)+B_{2} \exp \left(\delta_{2} z\right)
$$

where:

$$
\delta_{1}=-\frac{k}{\theta_{s 2}}\left[1+\sqrt{1+\theta_{s 2}^{2}\left(1+\frac{\bar{\Omega}_{2}}{k^{2}}-\frac{U_{p 2}^{2}}{C_{2}^{2}}\right)}\right]<0
$$




$$
\begin{gathered}
\delta_{2}=-\frac{k}{\theta_{s 2}}\left[1-\sqrt{1+\theta_{s 2}^{2}\left(1+\frac{\bar{\Omega}_{2}}{k^{2}}-\frac{U_{p}^{2}}{C_{2}^{2}}\right)}\right]>0, \\
\theta_{s 2}=\frac{2 k C_{s 2}^{2}}{g}=\frac{2 k C_{2}^{2}}{g} .
\end{gathered}
$$

Obviously, since the region of water is bounded along the vertical coordinate, both terms in (60) must be preserved for it.

\section{Waves of Wind}

Let us first consider the wind waves, when the amplitudes of pressure perturbations in air and water are determined by a system of expressions:

$$
\left\{\begin{array}{l}
P_{a 1}(z)=A \exp \left(\gamma_{1} z\right) \\
P_{a 2}(z)=B_{1} \exp \left(\delta_{1} z\right)+B_{2} \exp \left(\delta_{2} z\right)
\end{array}\right.
$$

Now it is necessary to determine the boundary condition connecting the perturbed pressures of two media on the water surface. Obviously, this condition must have the form

$$
\left.P_{2}\right|_{z=0}=\left.P_{1}\right|_{z=0}+\rho_{02} g \varsigma(x, t)
$$

where

$$
\xi(x, t)=a \exp [i(k x-\omega t)]
$$

is a displacement of the free surface of the liquid along the $Z$ axis, $a$ is the amplitude of the surface wave. Conditions for the continuity of the $z$ components of perturbed air and water velocities at the air-water interface will give:

$$
\left.V_{z 1}\right|_{z=0}=\left.V_{z 2}\right|_{z=0}=\frac{\partial \xi}{\partial t}
$$

and at the bottom of the water $(z=-H)$ we will have:

$$
\left.V_{z 2}\right|_{z=-H}=0 \text {. }
$$

By representing $V_{z}(x, z, t)$ from the first equation of the system (35) in the form $V_{z}(x, z, t)=V_{a}(z) \exp [i(k x-\omega t)]$, we obtain:

$$
V_{z}(x, z, t)=-\frac{i}{\rho_{0} \omega}\left[\frac{\mathrm{d} P_{a}(z)}{\mathrm{d} z}+\frac{g}{C^{2}} P_{a}(z)\right] \exp [i(k x-\omega t)] .
$$

Taking into account expressions (64) and (69), the boundary conditions (47), (49) and (50) will have the form

$$
\left\{\begin{array}{l}
A_{1}-B_{1}-B_{2}+\rho_{02} g a=0 \\
\frac{1}{\rho_{01} \omega}\left(\gamma_{1}+\frac{g}{\bar{C}_{1}^{2}}\right) A-\omega a=0 \\
\frac{1}{\rho_{02} \omega}\left(\delta_{1}+\frac{g}{C_{2}^{2}}\right) B_{1}+\frac{1}{\rho_{02} \omega}\left(\delta_{2}+\frac{g}{C_{2}^{2}}\right) B_{2}-\omega a=0 \\
\left(\delta_{1}+\frac{g}{C_{2}^{2}}\right) \exp \left(-\delta_{1} H\right) B_{1}+\left(\delta_{2}+\frac{g}{C_{2}^{2}}\right) \exp \left(-\delta_{2} H\right) B_{2}=0
\end{array}\right.
$$


By equating the determinant of the system (70) to zero, we obtain the dispersion equation for surface gravitational waves in the form

$$
\tilde{\delta}_{1} \exp \left(-\delta_{1} H\right)\left[\frac{\tilde{\gamma}_{1} \tilde{\delta}_{2} g}{\rho_{01} \omega^{2}}+\frac{\tilde{\delta}_{2}}{\rho_{02}}-\frac{\tilde{\gamma}_{1}}{\rho_{01}}\right]-\tilde{\delta}_{2} \exp \left(-\delta_{2} H\right)\left[\frac{\tilde{\gamma}_{1} \tilde{\delta}_{1} g}{\rho_{01} \omega^{2}}+\frac{\tilde{\delta}_{1}}{\rho_{02}}-\frac{\tilde{\gamma}_{1}}{\rho_{01}}\right]=0
$$

where:

$$
\begin{aligned}
& \tilde{\gamma}_{1}=\gamma_{1}+\frac{g}{\bar{C}_{1}^{2}}=\frac{2 g}{\bar{C}_{p 1}^{2}}+\frac{k}{\theta_{s 1}}\left[1-\sqrt{1+\theta_{s 1}^{2}\left(1+\frac{\bar{\Omega}_{1}}{k^{2}}-x^{2}\right)}\right], \\
& \tilde{\delta}_{1}=\delta_{1}+\frac{g}{\bar{C}_{2}^{2}}=\frac{k}{\theta_{s 2}}\left[1-\sqrt{1+\theta_{s 2}^{2}\left(1+\frac{\bar{\Omega}_{2}}{k^{2}}-\frac{\bar{C}_{1}^{2}}{C_{2}^{2}} x^{2}\right)}\right], \\
& \tilde{\delta}_{2}=\delta_{2}+\frac{g}{\bar{C}_{2}^{2}}=\frac{k}{\theta_{s 2}}\left[1+\sqrt{1+\theta_{s 2}^{2}\left(1+\frac{\bar{\Omega}_{2}}{k}-\frac{\bar{C}_{1}^{2}}{C_{2}^{2}} x^{2}\right)}\right] .
\end{aligned}
$$

Considering that $x \leq 1$, in formula (72) $x^{2}$ must be saved. As for the quantity $U_{p}^{2} / C_{2}^{2}=\left(\bar{C}_{1}^{2} / C_{2}^{2}\right) x^{2} \cong 0.03 x^{2}$ it is of the second or greater order of smallness and in the linear approximation should be discarded in formulas (73) and (74). For the minimum value $k_{\min }=9.05 \times 10^{-5} \mathrm{~m}^{-1}$ from (63) we have $\left(\theta_{s 2}\right)_{\min } \cong 40$. Thus, we can neglect the unit in comparison with $\theta_{s 2}$ and from (61), (62), (73) and (74) we have: $\delta_{1}=\tilde{\delta}_{1}=-k, \delta_{2}=\tilde{\delta}_{2}=k$. Then from (71) we easily obtain:

$$
\begin{aligned}
& \left\{\frac{2}{\theta_{p 1}}+\frac{1}{\vartheta_{s 1}}\left[1-\sqrt{1+\theta_{s 1}^{2}\left(1+\frac{\bar{\Omega}_{1}}{k^{2}}-x\right)}\right]\right\} \times\left[\frac{k g}{\omega^{2}} \tanh (k H)-1\right] \\
& -k \frac{\rho_{01}}{\rho_{02}} \tanh (k H)=0,
\end{aligned}
$$

where $\theta_{p 1}=2 k \bar{C}_{p 1}^{2} / g$. Equation (75) is a dispersion equation for wind waves and, as we see, it contains the thermodynamic parameters of both water and air. The last summand in (75) can be neglected due to its smallness, and then, it splits into two equations:

$$
\begin{gathered}
\frac{2}{\theta_{p 1}}+\frac{1}{\theta_{s 1}}\left[1-\sqrt{1+\theta_{s 1}^{2}\left(1+\frac{\bar{\Omega}_{1}}{k^{2}}-x^{2}\right)}\right]=0, \\
\frac{k g}{\omega^{2}} \tanh (k H)-1=0 .
\end{gathered}
$$

Equation (76) describes longitudinal waves in the air excited by perturbations on the surface of water and propagating along this surface. Let's consider this equation, ignoring the dependence of the sounds velocities on the coordinate $z$, i.e. take their values at sea level: $\bar{C}_{1}=C_{1}(0)=C_{s 1}(0)=340 \mathrm{~m} / \mathrm{sec}, C_{p 1}=\infty$. Then $\bar{\Omega}_{1}(z)=0$ and it will take the for

$$
1-\sqrt{1+\theta_{s 1}^{2}\left(1-x^{2}\right)}=0 \Rightarrow|x|=1 \Rightarrow\left|U_{p}\right|=C_{1}(0) .
$$

Obviously, in this case, the condition (57) is optional and the wave vector $k$ 
can take any value. We see that in this assumption, the speed of the wave in the air does not depend on the wavelength and is equal to the speed of sound at sea level. There is no doubt that perturbations whose wave lengths are comparable to the lengths of surface gravitational waves cannot propagate in the air at the speed of sound, and thus it is impossible to ignore the dependence of the speed of sound on the $z$ coordinate.

The solution to Equation (76) is:

$$
|x|=\sqrt{1+\frac{\vec{\Omega}_{1}}{k^{2}}-\frac{4}{\theta_{p 1} \theta_{s 1}}\left(1+\frac{\theta_{s 1}}{\theta_{p 1}}\right)}=\sqrt{1+\frac{1}{k^{2}}\left[\bar{\Omega}_{1}-\frac{g^{2}}{\bar{C}_{p 1}^{2} \bar{C}_{s 1}^{2}}\left(1+\frac{\bar{C}_{s 1}^{2}}{\bar{C}_{p 1}^{2}}\right)\right]} .
$$

Substituting in (79) the numerical values of the parameters from (48), we get

$$
|x|=\sqrt{1-\frac{1.17 \times 10^{-8}}{k^{2}}} \Rightarrow\left|U_{p}\right|=\sqrt{1-\frac{1.17 \times 10^{-8}}{k^{2}}} \bar{C}_{1} .
$$

From (80) we find

$$
k=\frac{1.08 \times 10^{-4}}{\sqrt{1-x^{2}}}
$$

which is consistent with the condition (57) and therefore the roots (79) are not extraneous for any valid values $k$. Table 1 shows the roots of Equation (62) for those values $k(\lambda)$, that satisfy condition (57)

We see that for $k>10^{-3} \mathrm{~m}^{-1} \Rightarrow \lambda<6.28 \times 10^{3} \mathrm{~m}$, the phase velocity of the wave in the air is constant and equal to $U_{p}=\bar{C}_{1} \cong 281.72 \mathrm{~m} / \mathrm{sec}$, and then, with a decrease of $k$, it falls and at $k=1.08 \times 10^{-4} \mathrm{~m}^{-1} \Rightarrow \lambda=5.80 \times 10^{4} \mathrm{~m}$, we have $x=0$, i.e., the wave stops. This means that at this wavelength, two regions of about $30 \mathrm{~km}$ long, with high and low pressures are formed in the atmosphere above water. In the area of low atmospheric pressure, the amplitude of the surface wave will increase and the pressure difference between the two areas will lead to the appearance of wind. When is further reduced $k$ to its minimum value, which is defined from (57) $k_{\text {min }} \cong 9 \times 10^{-5} \mathrm{~m}^{-1} \Rightarrow \lambda_{\text {max }} \cong 7 \times 10^{4} \mathrm{~m}$, the roots of Equation (76) and the corresponding frequencies of standing waves in the atmosphere become imaginary, which leads to a sharp increase in the pressure difference and, consequently, the amplitude of the surface wave and the wind force. This result clearly explains the reason for the drop in atmospheric pressure over the sea and ocean before the storm, as well as the reason for its strengthening.

Table 1. The dependence of the roots of Equation (76) on $k \geq 9.05 \times 10^{-5} \mathrm{~m}^{-1} \Rightarrow \lambda \leq 6.94 \times 10^{4} \mathrm{~m}$.

\begin{tabular}{cccccccc}
\hline$k\left(\mathrm{~m}^{-1}\right)$ & $10^{-1}$ & $10^{-2}$ & $10^{-3}$ & $1.10 \times 10^{-4}$ & $1.08 \times 10^{-4}$ & $1.06 \times 10^{-4}$ & $9.05 \times 10^{-5}$ \\
$\lambda(\mathrm{m})$ & 62.80 & 628.00 & 6280.00 & $5.71 \times 10^{4}$ & $5.81 \times 10^{4}$ & $5.92 \times 10^{4}$ & $6.94 \times 10^{4}$ \\
$X$ & 1.00 & 1.00 & 0.99 & 0.17 & 0.00 & $0.09 \mathrm{i}$ & $0.66 \mathrm{i}$ \\
$U_{p}(\mathrm{~m} / \mathrm{sec})$ & 281.72 & 281.72 & 278.90 & 47.89 & 0.00 & $25.35 \mathrm{i}$ & $186.59 \mathrm{i}$ \\
\hline
\end{tabular}


The solution to Equation (77), which describes surface gravitational waves on water, is:

$$
\left|U_{p}\right|=\sqrt{\frac{g}{k} \tanh (k H)}=\sqrt{\frac{g \lambda}{2 \pi} \tanh \left(\frac{2 \pi H}{\lambda}\right)}
$$

which coincides with (10) and thus, the existing theory for wind waves gives the correct result.

The phase velocity of a wave in the atmosphere does not depend on the depth of the ocean and decreases from the speed of sound to zero with increasing wavelength. In the ocean, on the contrary, the phase velocity increases from zero with increasing wavelength and depth. It is evident, that at certain wavelengths, which will depend on the depth of the ocean, these speeds will coincide and resonance of the frequencies will occur of waves in the atmosphere and the ocean. We can assume that this resonance is the cause of the "killer wave", especially since there is no other explanation yet. It should be noted, that the resonance alone is not enough for the appearance of a "killer wave"-it is essential that the oscillations in the air and in the water are occurring in antiphase.

The resonant wavelengths are obtained by equating the right sides of the Equations (80) and (82), i.e.

$$
\sqrt{1-\frac{1.17 \times 10^{-8} \lambda^{2}}{4 \pi^{2}}} \bar{C}_{1}=\sqrt{\frac{g \lambda}{2 \pi} \tanh \left(\frac{2 \pi H}{\lambda}\right)}
$$

For deep water $(2 \pi H / \lambda>1 \Rightarrow \tanh (2 \pi H / \lambda)=1)$, the Equation (83) obtains

$$
1-\frac{1.17 \times 10^{-8}}{4 \pi^{2}} \lambda^{2}=\frac{g}{2 \pi \bar{C}_{1}^{2}} \lambda
$$

The Equation (84) does not depend on $H$ and its solution is $\lambda \cong 6.4 \times 10^{4} \mathrm{~m}$ so therefore $H>10^{4} \mathrm{~m}$. Since there are practically no such depths with a flat relief, it can be said with great confidence that "killer waves" do not arise in deep water.

For the shallow water $(2 \pi H / \lambda<1 \Rightarrow \tanh (2 \pi H / \lambda)=2 \pi H / \lambda)$ we have

$$
\lambda=2 \pi \times 10^{4} \sqrt{\frac{1}{1.17}\left(1-\frac{g H}{\bar{C}_{1}^{2}}\right)}
$$

In order for the values $\lambda$ from (85) to satisfy the shallow water condition, the following condition must be fulfilled

$$
\left(1-\frac{g H}{\bar{C}_{1}^{2}}\right)>1.17 \times 10^{-8} H^{2}
$$

which obtains the following $H<5.3 \times 10^{3} \mathrm{~m}$. Therefore, the "killer waves" arise in shallow water, the depth of which does not exceed $5.3 \mathrm{~km}$.

The values of the killer wavelength for different depths and the corresponding values of the phase velocities, calculated by the formulas (85) and $U_{p}=\sqrt{g H}$ as well as the values of the periods and the ratio $2 \pi H / \lambda$ are given in Table 2 below. 
Table 2. Values of lengths, phase velocities and periods of the "killer wave" for various depths.

\begin{tabular}{ccccc}
\hline$H(\mathrm{~m})$ & $\lambda(\mathrm{m})$ & $U_{p}(\mathrm{~m} / \mathrm{sec})$ & $T(\mathrm{sec})$ & $2 \pi H / \lambda$ \\
\hline 200 & 57,331 & 44.3 & 1300 & 0.02 \\
400 & 56,595 & 62.6 & 904 & 0.04 \\
600 & 55,849 & 76.7 & 728 & 0.07 \\
800 & 55,092 & 88.5 & 622 & 0.09 \\
1000 & 54,325 & 99.0 & 549 & 0.11 \\
\hline
\end{tabular}

Figure 4 presents the graphs of the dependences of the phase velocities of longitudinal waves in the air (80) and on the water surface (82) for the same depths. We can see that the values of the resonant wavelengths coincide with the tabulated values.

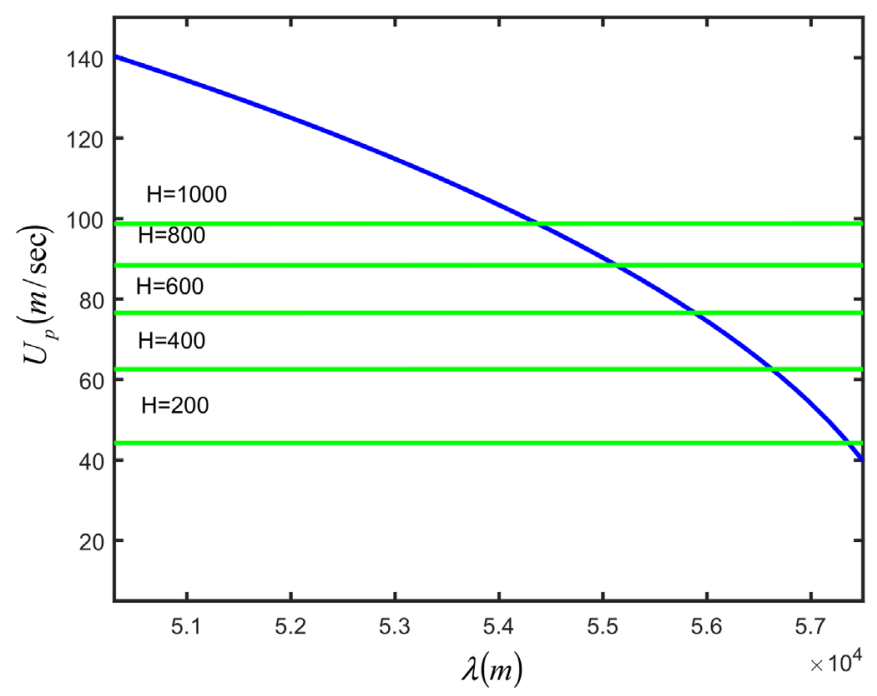

Figure 4. Plots of dependences of the phase velocities of longitudinal waves on the air (80) (blue curve) and on the water surface (82) (green curves) for different depths.

\section{Tsunami Waves}

Let us now consider tsunami waves, i.e. surface gravitational waves for those values $k$ that satisfy condition (58): $k<9.05 \times 10^{-5} \mathrm{~m}^{-1} \Rightarrow \lambda>6.90 \times 10^{4} \mathrm{~m}$. In this case, the amplitude of longitudinal waves in the atmosphere is determined by the expression (51), where $A_{1} \cong A_{2}<1$ and according to (52), (53) and (56), $\gamma_{1}<0$ and $\gamma_{2}<0$, i.e. both terms give the wave attenuation at $z \rightarrow \infty$. It can be seen that the wave corresponding to the first term decays faster than the wave corresponding to the second term, and therefore, to fulfill the condition of the wave's superficiality, it is sufficient to leave only the second term and thus we have:

$$
\left\{\begin{array}{l}
P_{a 1}(z)=A \exp \left(\gamma_{2} z\right) \\
P_{a 2}(z)=B_{1} \exp \left(\delta_{1} z\right)+B_{2} \exp \left(\delta_{2} z\right)
\end{array}\right.
$$


Note that boundary conditions (65), (67), and (68) are also valid for tsunami waves, and then, comparing (61) and (83), it is easy to verify that for tsunami waves we obtain an equation similar to Equation (71)

$$
\tilde{\delta}_{1} \exp \left(-\delta_{1} H\right)\left[\frac{\tilde{\gamma}_{2} \tilde{\delta}_{2} g}{\rho_{01} \omega^{2}}+\frac{\tilde{\delta}_{2}}{\rho_{02}}-\frac{\tilde{\gamma}_{2}}{\rho_{01}}\right]-\tilde{\delta}_{2} \exp \left(-\delta_{2} H\right)\left[\frac{\tilde{\gamma}_{2} \tilde{\delta}_{1} g}{\rho_{01} \omega^{2}}+\frac{\tilde{\delta}_{1}}{\rho_{02}}-\frac{\tilde{\gamma}_{2}}{\rho_{01}}\right]=0
$$

For waves, the length of which is $\lambda \geq 500 \mathrm{~km}$, the value $\theta_{s 2}$ changes in the interval $\theta_{s 2} \leq 5.5$ and, therefore, it cannot be neglected in comparison with the unit, in contrast to $\theta_{s 2}^{2}$. Then we will have:

$$
\left\{\begin{array}{l}
\tilde{\gamma}_{2}=\frac{2}{\theta_{p 1}}+\frac{1}{\theta_{s 1}}\left[1+\sqrt{1+\theta_{s 1}^{2}\left(1+\frac{\bar{\Omega}_{1}}{k^{2}}-x^{2}\right)}\right] \\
\delta_{1}=-\tilde{\delta}_{2}=-k\left(1+\frac{1}{\theta_{s 2}}\right), \delta_{2}=-\tilde{\delta}_{1}=k\left(1-\frac{1}{\theta_{s 2}}\right)
\end{array}\right.
$$

after which, Equation (88) takes the form

$$
\begin{aligned}
& \left\{\frac{2}{\theta_{p 1}}+\frac{1}{\vartheta_{s 1}}\left[1+\sqrt{1+\theta_{s 1}^{2}\left(1+\frac{\bar{\Omega}_{1}}{k^{2}}-x\right)}\right]\right\} \\
& \times\left[\left(\frac{k g}{\omega^{2}}+\frac{1}{\theta_{s 2}}\right) \tanh (k H)-1\right]-k \frac{\rho_{01}}{\rho_{02}} \tanh (k H)=0
\end{aligned}
$$

Ignoring the last term, Equation (90) again splits into two equations:

$$
\begin{gathered}
\frac{2}{\theta_{p 1}}+\frac{1}{\theta_{s 1}}\left[1+\sqrt{1+\theta_{s 1}^{2}\left(1+\frac{\bar{\Omega}_{1}}{k^{2}}-x^{2}\right)}\right]=0, \\
\left(\frac{k g}{\omega^{2}}+\frac{1}{\theta_{s 2}}\right) \tanh (k H)-1=0 .
\end{gathered}
$$

From condition (56) it follows that Equation (91) has no solution and this means that during a tsunami, wave processes in the atmosphere are not generated. As for Equation (92), its solution is

$$
U_{p}=\sqrt{\frac{(g / k) \tanh (k H)}{1-\tanh (k H) / \theta_{s 2}}}
$$

Considering that $k H<1 \Rightarrow \tanh (k H) \cong k H$, we will have:

$$
U_{p}=\sqrt{\frac{g H}{1-\frac{g H}{2 C_{2}^{2}}}} .
$$

For great depths, for example, at $H=10^{4} \mathrm{~m}$, the value $g H / 2 C_{2}^{2}=0.02$ and it can be ignored. Thus, the phase velocity of the tsunami wave is $U_{p}=\sqrt{g H}$.

\section{Conclusions}

This article does not claim to be highly accurate or to be the ultimate truth. The 
upper boundary of the troposphere changes depending on geographic parameters, and this concludes, that the average values of the problem parameters calculated here and, therefore, all the numerical data given in the article are rather conditional. However, undoubtedly the proposed method for solving the problem is new and makes it possible to trace the correlation between the ocean and the atmosphere during wave processes. In particular, it became clear why the atmospheric pressure in the ocean drops before the storm, as well as differentiating between the wavelengths of wind and tsunami became possible.

Its apparent advantage is also that at the level of a highly plausible hypothesis, it reveals the greatest mystery of nature called the "Killer Wave". Now it is clear why this wave is solitary. This is due to the fact that the flat topography of the ocean floor is disturbed at the distances of the order of the wavelengths that we calculated. It is also clear why a cavity, is formed before the wave. This is attributed to the fact that there is a region in front of the wave, where the pressure in the water sharply drops and in the atmosphere sharply increases.

All this became possible after the discovery of the isobaric speed of sound and the dependence of the true value of the speed of sound in the atmosphere on the vertical coordinate. This led to a radical change in many established dogmas and ideas in aero and hydrodynamics, which are recognized by the international scientific community (work [8] is posted on several sites on the Internet (see, for example, [16]) and work [14] is posted in the NASA database [17]). Despite this, the Internet [18] to this day gives the values of the speed of sound at different heights of the atmosphere, calculated by the formula (15). Obviously, this can be explained by the fact that our theoretical results have not been experimentally confirmed. We hope that this article will be able to help popularize this problem and in the relevant scientific community will show desire in conducting the necessary experiments. The importance of such experiments also lies in the fact that if it is possible in laboratories to create conditions corresponding to the upper boundary of the troposphere and to confirm the fact of heat release, we will get an alternative source of energy.

\section{Conflicts of Interest}

The authors declare no conflicts of interest regarding the publication of this paper.

\section{References}

[1] Kirtskhalia, V.G. (2016) Journal of Fluids, 2016, Article ID: 4519201. https://doi.org/10.1155/2016/4519201

[2] Landau, L.D. and Lifchitz, E.N. (1988) Theoretical Physics, Hydrodynamics. Vol. 6, Nauka, Moscow.

[3] Kowalik, Z. (2012) Introduction to Numerical Modeling of Tsunami Waves. Institute of Marine Science University of Alaska, Fairbank. https://www.sfos.uaf.edu/directory/faculty/kowalik/Tsunami Book

[4] Stoker, J.J. (1957) Water Waves. Interscience, New York. 
[5] Whitham, G. (1974) Linear and Nonlinear Waves. John Wiley \& Sons (Wiley-Interscience), New York.

[6] Gossard, E.E. and Hooke, W.H. (1975) Waves in the Atmosphere.

[7] Wang, G.S.K. (1986) The Journal of the Acoustical Society of America, 79, 1359-1366.

[8] Kirtskhalia, V.G. (2012) Open Journal of Acoustics, 2, 80-85. https://doi.org/10.4236/oja.2012.22009

[9] Kirtskhalia, V. (2012) Open Journal of Acoustics, 2, 115-120. https://doi.org/10.4236/oja.2012.23013

[10] National Aeronautics and Space Administration (1976) U.S. Standard Atmosphere.

[11] Kirtskhalia, V.G. (2021) IOP Conference Series. Materials Science and Engineering, 1024, Article ID: 012037. https://doi.org/10.1088/1757-899X/1024/1/012037

[12] Sorokhtin, O.G. (2009) The Process of Absorption of Ultra-Violet Radiation of the Sun Terrestrial Atmosphere. Bulletin of the Russian Academy of Natural Sciences, $2009 / 3$.

[13] Kirtskhalia, V.G. (2015) Journal of Modern Physics, 7, 948-954. https://doi.org/10.4236/jmp.2015.67099

[14] Kirtskhalia, V.G. (2013) Journal of Modern Physics, 4, 1075-1079. https://doi.org/10.4236/jmp.2013.48144

[15] Kirtskhalia, V.G. (2019) Journal of Modern Physics, 10, 452-458. https://doi.org/10.4236/jmp.2019.104030

[16] Kirtskhalia, V.G. (2012) Open Journal of Acoustics, 2, 80-85. https://www.researchgate.net/publication/274750587 Speed of SoundinAtmospher eof the Earth

[17] The Smithsonian/NASA Astrophysics Date System.

[18] http://www.sengpielaudio.com/calculator-speedsound.htm 